\title{
Positive solutions for an impulsive boundary value problem with Caputo fractional derivative
}

Keyu Zhang ${ }^{\mathrm{a}, \mathrm{b}}$, Jiafa $\mathrm{Xu}^{\mathrm{c}, *}$

a School of Mathematics, Shandong University, Jinan, Shandong, 250100, P. R. China.

${ }^{b}$ Department of Mathematics, Qilu Normal University, Jinan, Shandong, 250013, P. R. China.

${ }^{\prime}$ School of Mathematical Sciences, Chongqing Normal University, Chongqing 401331, P. R. China.

Communicated by J. Brzdek

\begin{abstract} impulsive boundary value problem with Caputo fractional derivative

$$
\left\{\begin{array}{l}
{ }^{c} D_{t}^{q} u(t)=f(t, u(t)), \quad \text { a.e. } t \in[0,1] ; \\
\Delta u\left(t_{k}\right)=I_{k}\left(u\left(t_{k}\right)\right), \quad \Delta u^{\prime}\left(t_{k}\right)=J_{k}\left(u\left(t_{k}\right)\right), \quad k=1,2, \ldots, m ; \\
a u(0)-b u(1)=0, \quad a u^{\prime}(0)-b u^{\prime}(1)=0,
\end{array}\right.
$$
\end{abstract}

In this work we use fixed point theorem method to discuss the existence of positive solutions for the

where $q \in(1,2)$ is a real number, $a, b$ are real constants with $a>b>0$, and ${ }^{c} D_{t}^{q}$ is the Caputo's fractional derivative of order $q, f:[0,1] \times \mathbb{R}^{+} \rightarrow \mathbb{R}^{+}$and $I_{k}, J_{k}: \mathbb{R}^{+} \rightarrow \mathbb{R}^{+}$are continuous functions, $k=1,2, \ldots, m$, $\mathbb{R}^{+}:=[0,+\infty)$. (C)2016 All rights reserved.

Keywords: Caputo fractional derivative, impulsive boundary value problem, fixed point theorem, positive solution.

2010 MSC: 34B10, 34B15, 34B37.

\section{Introduction}

In this work we study the impulsive boundary value problem with Caputo fractional derivative

$$
\left\{\begin{array}{l}
{ }^{c} D_{t}^{q} u(t)=f(t, u(t)), \quad \text { a.e. } t \in[0,1] \\
\Delta u\left(t_{k}\right)=I_{k}\left(u\left(t_{k}\right)\right), \quad \Delta u^{\prime}\left(t_{k}\right)=J_{k}\left(u\left(t_{k}\right)\right), \quad k=1,2, \ldots, m ; \\
a u(0)-b u(1)=0, \quad a u^{\prime}(0)-b u^{\prime}(1)=0,
\end{array}\right.
$$

\footnotetext{
${ }^{*}$ Corresponding author

Email addresses: keyu_292@163.com (Keyu Zhang), xujiafa292@sina.com (Jiafa Xu)
} 
where $q \in(1,2)$ is a real number, $a, b$ are real constants with $a>b>0$, and ${ }^{c} D_{t}^{q}$ is the Caputo's fractional derivative of order $q ; t_{k}(k=1,2, \ldots, m, m \geq 1$ is a fixed integer $)$ are constants with $0=t_{0}<t_{1}<\cdots<$ $t_{m}<t_{m+1}=1, u\left(t_{k}^{+}\right)=\lim _{h \rightarrow 0} u\left(t_{k}+h\right)$ and $u\left(t_{k}^{-}\right)=\lim _{h \rightarrow 0} u\left(t_{k}-h\right)$ represent the right-hand and left-hand limits of $u(t)$ at $t=t_{k}$, respectively. Moreover, $f, I_{k}$, and $J_{k}$ satisfy the condition:

(H1). $f:[0,1] \times \mathbb{R}^{+} \rightarrow \mathbb{R}^{+}$and $I_{k}, J_{k}: \mathbb{R}^{+} \rightarrow \mathbb{R}^{+}(k=1,2, \ldots, m)$ are continuous functions.

Denote $J=[0,1], J_{0}=\left[0, t_{1}\right], J_{k}=\left(t_{k}, t_{k+1}\right](k=1,2, \ldots, m)$. Furthermore, we define

$P C(J)=\left\{u \mid u: J \rightarrow \mathbb{R}\right.$ is continuous at $\neq t_{k}$, and $u\left(t_{k}^{+}\right), u\left(t_{k}^{-}\right)$exist, $\left.u\left(t_{k}^{-}\right)=u\left(t_{k}\right), k=1,2, \ldots, m\right\}$.

Clearly, $P C(J)$ is a Banach space with the norm $\|u\|=\sup _{t \in J}|u(t)|$ for $u \in P C(J)$. Note that $C(J)$, which represents the set of all continuous functions on $J$, is also a Banach space with $\|u\|$.

As is well known, it is an important method to express the solutions of differential equations by Green's function. However, for impulsive differential equations of fractional order (see [1, 5, 9, 11, 14, 15, 17, 19] and the references therein), their integral forms are very complicated, and cannot be formulated by virtue of some suitable Green's functions. For example, in [14, Wang, Ahmad and Zhang investigated the existence and uniqueness of solutions for a mixed boundary value problem of fractional differential equations with impulses

$$
\left\{\begin{array}{l}
{ }^{c} D^{\alpha} u(t)=f(t, u(t)), \quad 1<\alpha \leq 2, \quad t \in J^{\prime} ; \\
\Delta u\left(t_{k}\right)=I_{k}\left(u\left(t_{k}\right)\right), \quad \Delta u^{\prime}\left(t_{k}\right)=I_{k}^{*}\left(u\left(t_{k}\right)\right), \quad k=1,2, \ldots, p ; \\
T u^{\prime}(0)=-a u(0)-b u(T), \quad T u^{\prime}(T)=c u(0)+d u(T),
\end{array}\right.
$$

which can be written in the form

$$
\begin{aligned}
u(t)= & \int_{t_{k}}^{t} \frac{(t-s)^{\alpha-1}}{\Gamma(\alpha)} f(s, u(s)) \mathrm{d} s+\lambda_{1}(t) \int_{t_{p}}^{T} \frac{(T-s)^{\alpha-1}}{\Gamma(\alpha)} f(s, u(s)) \mathrm{d} s \\
& -\lambda_{2}(t) \int_{t_{p}}^{T} \frac{(T-s)^{\alpha-2}}{\Gamma(\alpha-1)} f(s, u(s)) \mathrm{d} s+\sum_{i=1}^{k}\left[\int_{t_{i-1}}^{t_{i}} \frac{\left(t_{i}-s\right)^{\alpha-1}}{\Gamma(\alpha)} f(s, u(s)) \mathrm{d} s+I_{i}\left(u\left(t_{i}\right)\right)\right] \\
& +\sum_{i=1}^{k-1}\left(t_{k}-t_{i}\right)\left[\int_{t_{i-1}}^{t_{i}} \frac{\left(t_{i}-s\right)^{\alpha-2}}{\Gamma(\alpha-1)} f(s, u(s)) \mathrm{d} s+I_{i}^{*}\left(u\left(t_{i}\right)\right)\right] \\
& +\sum_{i=1}^{k}\left(t-t_{k}\right)\left[\int_{t_{i-1}}^{t_{i}} \frac{\left(t_{i}-s\right)^{\alpha-2}}{\Gamma(\alpha-1)} f(s, u(s)) \mathrm{d} s+I_{i}^{*}\left(u\left(t_{i}\right)\right)\right] \\
& +\lambda_{1}(t) \sum_{i=1}^{p}\left[\int_{t_{i-1}}^{t_{i}} \frac{\left(t_{i}-s\right)^{\alpha-1}}{\Gamma(\alpha)} f(s, u(s)) \mathrm{d} s+I_{i}\left(u\left(t_{i}\right)\right)\right] \\
& +\lambda_{1}(t) \sum_{i=1}^{p}\left(t_{p}-t_{i}\right)\left[\int_{t_{i-1}}^{t_{i}} \frac{\left(t_{i}-s\right)^{\alpha-2}}{\Gamma(\alpha-1)} f(s, u(s)) \mathrm{d} s+I_{i}^{*}\left(u\left(t_{i}\right)\right)\right] \\
& -\sum_{i=1}^{p}\left[\lambda_{3}(t)+\lambda_{1}(t) t_{p}\right]\left[\int_{t_{i-1}}^{t_{i}} \frac{\left(t_{i}-s\right)^{\alpha-2}}{\Gamma(\alpha-1)} f(s, u(s)) \mathrm{d} s+I_{i}^{*}\left(u\left(t_{i}\right)\right)\right] .
\end{aligned}
$$

We all know that impulsive differential equations with integer order can be expressed by Green's function (see for example [7, 16, 20]), therefore, it is a natural problem whether or not the same result holds for the fractional order case. To the best of our knowledge, only [10, 21 23] are devoted to this direction. In [10], Liu and Jia considered the fractional impulsive differential equations:

$$
\left\{\begin{array}{l}
{ }^{c} D_{0+}^{\alpha} u(t)=f\left(t, u(t), \quad{ }^{c} D_{0+}^{\beta} u(t)\right), \quad t \in J^{\prime} ; \\
\Delta u\left(t_{k}\right)=I_{k}\left(u\left(t_{k}\right), \quad{ }^{c} D_{0+}^{\beta} u\left(t_{k}\right)\right) ; \\
\Delta^{c} D_{0+}^{\beta} u\left(t_{k}\right)=Q_{k}\left(u\left(t_{k}\right), \quad{ }^{c} D_{0+}^{\beta} u\left(t_{k}\right)\right), \quad k=1,2, \ldots, m ; \\
u(0)=0, \quad u(1)=\int_{0}^{1} u(t) g(t) \mathrm{d} t,
\end{array}\right.
$$


which can be expressed by

$$
\begin{aligned}
u(t)= & \int_{0}^{1} G(t, s) f\left(s, u(s),{ }^{c} D_{0+}^{\beta} u(s)\right) \mathrm{d} s+\sum_{i=1}^{m} H\left(t, t_{i}\right) I_{i}\left(u\left(t_{i}\right),{ }^{c} D_{0+}^{\beta} u\left(t_{i}\right)\right) \\
& +\sum_{i=1}^{m} K\left(t, t_{i}\right) Q_{i}\left(u\left(t_{i}\right),{ }^{c} D_{0+}^{\beta} u\left(t_{i}\right)\right) .
\end{aligned}
$$

By Schauder fixed point theorem and Krasnoselskii fixed point theorem, they established some existence theorems for the above problem.

Inspired by the above mentioned works, in this paper by Green's function and fixed point theorem method, we obtain the existence of (positive) solutions for (1.1) with the assumptions that the growth of $f$ is superlinear, asymptotically linear and sublinear.

\section{Preliminaries}

Let us recall some notations and preliminary lemmas of fractional calculus, for more details, see [12, 13].

Definition 2.1. The Riemann-Liouville fractional integral operator of order $\alpha>0$, of function $f$ : $(0,+\infty) \rightarrow(-\infty,+\infty)$ is defined as

$$
I_{0+}^{\alpha} f(t)=\frac{1}{\Gamma(\alpha)} \int_{0}^{t}(t-s)^{\alpha-1} f(s) \mathrm{d} s,
$$

where $\Gamma(\cdot)$ is the Euler gamma function.

Definition 2.2. The fractional derivative of $f$ in the Caputo sense is defined as

$$
{ }^{c} D_{t}^{\alpha} f(t)=\frac{1}{\Gamma(n-\alpha)} \int_{0}^{t}(t-s)^{n-\alpha-1} f^{(n)}(s) \mathrm{d} s, \quad n-1<\alpha<n,
$$

where $n=[\alpha]+1,[\alpha]$ denotes the integer part of the number $\alpha$.

Lemma 2.3. Let $\alpha>0$. Then the differential equation ${ }^{c} D_{t}^{\alpha} u(t)=0$ has a unique solution

$$
u(t)=c_{0}+c_{1} t+\cdots+c_{n-1} t^{n-1}
$$

for some $c_{i} \in \mathbb{R}(i=0,1, \ldots, n-1)$, where $n=[\alpha]+1$.

Lemma 2.4. Assume that $u \in C(0,1) \cap L(0,1)$ with a derivative of order $\alpha>0$ that belongs to $C(0,1) \cap$ $L(0,1)$. Then

$$
I_{0+}^{\alpha}{ }^{c} D_{t}^{\alpha} u(t)=u(t)+c_{0}+c_{1} t+\cdots+c_{n-1} t^{n-1}
$$

for some $c_{i} \in \mathbb{R}(i=0,1, \ldots, n-1)$, where $n=[\alpha]+1$.

Lemma $2.5([22$, Lemma 2.5]). Let $y \in C(J)$. Then the unique solution of the boundary value problem

$$
\left\{\begin{array}{l}
{ }^{c} D_{t}^{q} u(t)=y(t), \quad \text { a.e. } t \in[0,1] \\
\Delta u\left(t_{k}\right)=I_{k}\left(u\left(t_{k}\right)\right), \quad \Delta u^{\prime}\left(t_{k}\right)=J_{k}\left(u\left(t_{k}\right)\right), \quad k=1,2, \ldots, m ; \\
a u(0)-b u(1)=0, \quad a u^{\prime}(0)-b u^{\prime}(1)=0,
\end{array}\right.
$$

is given by

$$
u(t)=\int_{0}^{1} G_{1}(t, s) y(s) \mathrm{d} s+\sum_{i=1}^{m} G_{2}\left(t, t_{i}\right) J_{i}\left(u\left(t_{i}\right)\right)+\sum_{i=1}^{m} G_{3}\left(t, t_{i}\right) I_{i}\left(u\left(t_{i}\right)\right)
$$


where

$$
\begin{aligned}
& G_{1}(t, s)=\left\{\begin{array}{l}
\frac{(t-s)^{q-1}}{\Gamma(q)}+\frac{b(1-s)^{q-1}}{(a-b) \Gamma(q)}+\frac{b(q-1) t(1-s)^{q-2}}{(a-b) \Gamma(q)}+\frac{b^{2}(q-1)(1-s)^{q-2}}{(a-b)^{2} \Gamma(q)}, \quad 0 \leq s \leq t \leq 1 ; \\
\frac{b(1-s)^{q-1}}{(a-b) \Gamma(q)}+\frac{b(q-1) t(1-s)^{q-2}}{(a-b) \Gamma(q)}+\frac{b^{2}(q-1)(1-s)^{q-2}}{(a-b)^{2} \Gamma(q)}, \quad 0 \leq t \leq s \leq 1,
\end{array}\right. \\
& G_{2}(t, s)= \begin{cases}\frac{a b}{(a-b)^{2}}+\frac{a\left(t-t_{i}\right)}{a-b}, \quad 0 \leq t_{i}<t \leq 1, \quad i=1,2, \ldots, m ; \\
\frac{a b}{(a-b)^{2}}+\frac{b\left(t-t_{i}\right)}{a-b}, \quad 0 \leq t \leq t_{i} \leq 1, \quad i=1,2, \ldots, m,\end{cases} \\
& G_{3}(t, s)= \begin{cases}\frac{a}{a-b}, \quad 0 \leq t_{i}<t \leq 1, \quad i=1,2, \ldots, m ; \\
\frac{b}{a-b}, \quad 0 \leq t \leq t_{i} \leq 1, \quad i=1,2, \ldots, m .\end{cases}
\end{aligned}
$$

Lemma 2.6 ([22, Lemma 2.6]). Let $a, b$ be real constants with $a>b>0$. Then $G_{i}(i=1,2,3)$ have the following properties

(i) $G_{1}(t, s) \in C\left(J \times J, \mathbb{R}^{+}\right)$and $G_{1}(t, s)>0, G_{2}\left(t, t_{i}\right)>0, G_{3}\left(t, t_{i}\right)>0$ for all $t, t_{i}, s \in(0,1)$,

(ii) there exists a negative function $M(s), s \in[0,1]$ such that

$$
\frac{b}{a} M(s) \leq G_{1}(t, s) \leq M(s)
$$

where

$$
M(s)=\frac{a[(1-s) a-(2-s-q) b](1-s)^{q-2}}{(a-b)^{2} \Gamma(q)}, \quad s \in[0,1]
$$

(iii)

$$
\frac{b^{2}}{(a-b)^{2}} \leq G_{2}\left(t, t_{i}\right) \leq \frac{a^{2}}{(a-b)^{2}}, \quad \frac{b}{a-b} \leq G_{3}\left(t, t_{i}\right) \leq \frac{a}{a-b}, \quad \forall t, t_{i} \in[0,1] .
$$

For convenience, we need to calculate the following integral

$$
\kappa_{1}:=\int_{0}^{1} M(s) \mathrm{d} s=\frac{a^{2}(q-1)+a b q(q-2)+a b}{q(q-1)(a-b)^{2} \Gamma(q)} .
$$

We define the operator $\mathcal{A}: P C(J) \rightarrow P C(J)$ by

$$
(\mathcal{A} u)(t):=\int_{0}^{1} G_{1}(t, s) f(s, u(s)) \mathrm{d} s+\sum_{i=1}^{m} G_{2}\left(t, t_{i}\right) J_{i}\left(u\left(t_{i}\right)\right)+\sum_{i=1}^{m} G_{3}\left(t, t_{i}\right) I_{i}\left(u\left(t_{i}\right)\right),
$$

where $G_{i}(i=1,2,3)$ are defined in (2.3), (2.4) and (2.5). Then from Lemma 2.5, solving the solutions of (1.1) reduces to solve the fixed points of the operator equation $u=\mathcal{A} u$. Furthermore, we can adopt the Ascoli-Arzela theorem to prove $\mathcal{A}$ is a completely continuous operator.

Define $P=\{u \in P C(J): u(t) \geq 0, t \in[0,1]\}$, and $P_{0}=\left\{u \in P C(J): u(t) \geq \frac{b^{2}}{a^{2}}\|u\|, t \in[0,1]\right\}$. Then $P, P_{0}$ are cone on $P C(J)$. Moreover, we easily obtain the following lemma.

Lemma 2.7. $\mathcal{A}(P) \subset P_{0}$.

Let $E$ be a Banach space, $P$ be a cone on $E$, and $B_{R}:=\{u \in E:\|u\|<R\}$ for $R>0$ in the sequel.

Lemma 2.8 ([6]). Let $\mathcal{A}: \bar{B}_{R} \cap P \rightarrow P$ be a completely continuous operator. If there exists $v_{0} \in P \backslash\{0\}$ such that $v-\mathcal{A} v \neq \lambda v_{0}$ for all $v \in \partial B_{R} \cap P$ and $\lambda \geq 0$, then $i\left(\mathcal{A}, B_{R} \cap P, P\right)=0$, where $i$ is the fixed point index on $P$. 
Lemma $2.9([6])$. Let $\mathcal{A}: \bar{B}_{R} \cap P \rightarrow P$ be a completely continuous operator. If $v \neq \lambda \mathcal{A} v$ for all $v \in \partial B_{R} \cap P$ and $0 \leq \lambda \leq 1$, then $i\left(\mathcal{A}, B_{R} \cap P, P\right)=1$.

Lemma $2.10([8])$. Let $\mathcal{A}: E \rightarrow E$ be a completely continuous operator. Assume that $T: E \rightarrow E$ is a bounded linear operator such that 1 is not an eigenvalue of $T$ and

$$
\lim _{\|u\| \rightarrow \infty} \frac{\|\mathcal{A} u-T u\|}{\|u\|}=0 .
$$

Then $\mathcal{A}$ has a fixed point in $E$.

\section{Main results}

Theorem 3.1. Assume that

(H2). $f:[0,1] \times \mathbb{R} \rightarrow \mathbb{R}$ and $I_{k}, J_{k}: \mathbb{R} \rightarrow \mathbb{R}(k=1,2, \ldots, m)$ are continuous functions, moreover,

$$
\lim _{u \rightarrow \infty} \frac{f(t, u)}{u}=\lambda, \quad \text { uniformly int } \in[0,1],
$$

and

$$
\lim _{u \rightarrow \infty} \frac{I_{k}(u)}{u}=\lambda, \lim _{u \rightarrow \infty} \frac{J_{k}(u)}{u}=\lambda, k=1,2, \ldots, m
$$

If

$$
|\lambda|<\left[\kappa_{1}+m\left(\frac{a^{2}}{(a-b)^{2}}+\frac{a}{a-b}\right)\right]^{-1}
$$

then (1.1) has a nontrivial solution when $f(t, 0) \not \equiv 0$ for $t \in[0,1]$.

Proof. Define $T: P C(J) \rightarrow P C(J)$ by

$$
(T u)(t):=\lambda\left[\int_{0}^{1} G_{1}(t, s) u(s) \mathrm{d} s+\sum_{i=1}^{m} G_{2}\left(t, t_{i}\right) u\left(t_{i}\right)+\sum_{i=1}^{m} G_{3}\left(t, t_{i}\right) u\left(t_{i}\right)\right] .
$$

Then $T$ is a bounded linear operator. From Lemma 2.5, equation (3.1) is equivalent to

$$
\left\{\begin{array}{l}
{ }^{c} D_{t}^{q} u(t)=\lambda u(t), \quad \text { a.e. } t \in[0,1] ; \\
\Delta u\left(t_{k}\right)=\lambda u\left(t_{k}\right), \quad \Delta u^{\prime}\left(t_{k}\right)=\lambda u\left(t_{k}\right), \quad k=1,2, \ldots, m ; \\
a u(0)-b u(1)=0, \quad a u^{\prime}(0)-b u^{\prime}(1)=0 .
\end{array}\right.
$$

Next, we consider the following two cases.

Case 1. $\lambda=0$. Equation $(3.2)$ is a problem without impulse, and from Lemma 2.3 we have

$$
u(t)=c_{0}+c_{1} t
$$

for some $c_{i} \in \mathbb{R}, i=0,1$. In view of the boundary conditions 3.2 , we have $c_{0}=c_{1}=0$ and thus $u(t) \equiv 0$ for $t \in[0,1]$. This shows 3.2 has only a trivial solution.

Case 2. $\lambda \neq 0$. From Case 1 we see 3.2 has nontrivial solutions. Let $u$ be a nontrivial solution for $(3.2)$ and then $\|u\|>0$. Suppose that 1 is an eigenvalue of $T$. Then we have

$$
\begin{aligned}
\|u\| & =\|T u\| \leq|\lambda|\|u\|\left[\int_{0}^{1} G_{1}(t, s) \mathrm{d} s+\sum_{i=1}^{m} G_{2}\left(t, t_{i}\right)+\sum_{i=1}^{m} G_{3}\left(t, t_{i}\right)\right] \\
& \leq|\lambda|\left[\kappa_{1}+m\left(\frac{a^{2}}{(a-b)^{2}}+\frac{a}{a-b}\right)\right]\|u\|<\|u\| .
\end{aligned}
$$


This is impossible.

To sum up, 1 is not an eigenvalue of $T$.

From (H2), for all $\varepsilon>0$, there exists $M>0$ such that

$$
|f(t, u)-\lambda u| \leq \varepsilon|u|,\left|I_{k}(u)-\lambda u\right| \leq \varepsilon|u|,\left|J_{k}(u)-\lambda u\right| \leq \varepsilon|u|, \text { for } t \in[0,1],|u| \geq M .
$$

Moreover, if $|u| \leq M$, then $|f(t, u)-\lambda u|,\left|I_{k}(u)-\lambda u\right|$ and $\left|J_{k}(u)-\lambda u\right|$ are bounded. Hence, there exists $M_{1}>0$ such that

$$
|f(t, u)-\lambda u| \leq \varepsilon|u|+M_{1},\left|I_{k}(u)-\lambda u\right| \leq \varepsilon|u|+M_{1},\left|J_{k}(u)-\lambda u\right| \leq \varepsilon|u|+M_{1}, \text { for } t \in[0,1], u \in \mathbb{R} .
$$

Hence

$$
\begin{aligned}
\|\mathcal{A} u-T u\|= & \sup _{t \in[0,1]} \mid \int_{0}^{1} G_{1}(t, s)[f(s, u(s))-\lambda u(s)] \mathrm{d} s \\
& +\sum_{i=1}^{m} G_{2}\left(t, t_{i}\right)\left[J_{i}\left(u\left(t_{i}\right)\right)-\lambda u\left(t_{i}\right)\right]+\sum_{i=1}^{m} G_{3}\left(t, t_{i}\right)\left[I_{i}\left(u\left(t_{i}\right)\right)-\lambda u\left(t_{i}\right)\right] \mid \\
\leq & \sup _{t \in[0,1]} \int_{0}^{1} G_{1}(t, s)|f(s, u(s))-\lambda u(s)| \mathrm{d} s \\
& +\sup _{t \in[0,1]} \sum_{i=1}^{m} G_{2}\left(t, t_{i}\right)\left|J_{i}\left(u\left(t_{i}\right)\right)-\lambda u\left(t_{i}\right)\right|+\sup _{t \in[0,1]} \sum_{i=1}^{m} G_{3}\left(t, t_{i}\right)\left|I_{i}\left(u\left(t_{i}\right)\right)-\lambda u\left(t_{i}\right)\right| \\
\leq & \left(\varepsilon\|u\|+M_{1}\right)\left[\kappa_{1}+m\left(\frac{a^{2}}{(a-b)^{2}}+\frac{a}{a-b}\right)\right]
\end{aligned}
$$

which implies that

$$
\lim _{\|u\| \rightarrow \infty} \frac{\|\mathcal{A} u-T u\|}{\|u\|} \leq \lim _{\|u\| \rightarrow \infty} \frac{\left(\varepsilon\|u\|+M_{1}\right)\left[\kappa_{1}+m\left(\frac{a^{2}}{(a-b)^{2}}+\frac{a}{a-b}\right)\right]}{\|u\|}=\varepsilon\left[\kappa_{1}+m\left(\frac{a^{2}}{(a-b)^{2}}+\frac{a}{a-b}\right)\right] .
$$

Note that the arbitrariness of $\varepsilon$, so

$$
\lim _{\|u\| \rightarrow \infty} \frac{\|\mathcal{A} u-T u\|}{\|u\|}=0 .
$$

Therefore, from Lemma 2.10, $\mathcal{A}$ has a fixed point in $P C(J)$, that is, 1.1 has at least one solution $u$. Further, we can assert that $u$ is nontrivial when $f(t, 0) \not \equiv 0$ for $t \in[0,1]$. This completes the proof.

In order to establish the following two theorems, we need some conditions as follows:

(H3). There exist $c>0$ and $a_{1} \geq 0, a_{2} \geq 0, a_{3} \geq 0$ satisfying

$$
a_{3} b^{4} m+a_{2} b^{3}(a-b) m>(a-b)^{2}\left(a^{2}-a b a_{1} \kappa_{1}\right)
$$

such that

$$
f(t, u) \geq a_{1} u-c, I_{k}(u) \geq a_{2} u-c, J_{k}(u) \geq a_{3} u-c, \text { for all } t \in[0,1], u \in \mathbb{R}^{+} \text {. }
$$

(H4). There exist $r>0$ and $b_{1} \geq 0, b_{2} \geq 0, b_{3} \geq 0$ satisfying

$$
\left(1-\kappa_{1} b_{1}\right) b^{2}(a-b)^{2}>m\left[a^{4} b_{3}+a^{3}(a-b) b_{2}\right]
$$

such that

$$
f(t, u) \leq b_{1} u, I_{k}(u) \leq b_{2} u, J_{k}(u) \leq b_{3} u, \text { for all } t \in[0,1], u \in[0, r] .
$$


(H5). There exist $r>0$ and $a_{4} \geq 0, a_{5} \geq 0, a_{6} \geq 0$ satisfying

$$
a_{6} b^{4} m+a_{5} b^{3}(a-b) m>(a-b)^{2}\left(a^{2}-a b a_{4} \kappa_{1}\right)
$$

such that

$$
f(t, u) \geq a_{4} u, I_{k}(u) \geq a_{5} u, J_{k}(u) \geq a_{6} u, \text { for all } t \in[0,1], u \in[0, r] .
$$

(H6). There exist $c>0$ and $b_{4} \geq 0, b_{5} \geq 0, b_{6} \geq 0$ satisfying

$$
\left(1-\kappa_{1} b_{4}\right) b^{2}(a-b)^{2}>m\left[a^{4} b_{6}+a^{3}(a-b) b_{5}\right]
$$

such that

$$
f(t, u) \leq b_{4} u+c, I_{k}(u) \leq b_{5} u+c, J_{k}(u) \leq b_{6} u+c, \text { for all } t \in[0,1], u \in \mathbb{R}^{+} .
$$

Theorem 3.2. Suppose that (H1), (H3) and (H4) hold. Then (1.1) has at least one positive solution.

Proof. Let $\mathcal{M}_{1}=\{u \in P: u=\mathcal{A} u+\lambda \psi, \lambda \geq 0\}$, where $\psi \in P_{0}$ is a given element. From Lemma 2.7. $u \in \mathcal{M}_{1}$ implies that $u \in P_{0}$. We shall prove that $\mathcal{M}_{1}$ is bounded. If $u \in \mathcal{M}_{1}$, then $u \geq \mathcal{A} u$. This shows

$$
u(t) \geq \int_{0}^{1} G_{1}(t, s) f(s, u(s)) \mathrm{d} s+\sum_{i=1}^{m} G_{2}\left(t, t_{i}\right) J_{i}\left(u\left(t_{i}\right)\right)+\sum_{i=1}^{m} G_{3}\left(t, t_{i}\right) I_{i}\left(u\left(t_{i}\right)\right) .
$$

Multiplying by $M(t)$ on both sides of the above and integrating over [0,1], we obtain

$$
\begin{aligned}
\int_{0}^{1} u(t) M(t) \mathrm{d} t & \geq \int_{0}^{1} M(t)\left[\int_{0}^{1} G_{1}(t, s) f(s, u(s)) \mathrm{d} s+\sum_{i=1}^{m} G_{2}\left(t, t_{i}\right) J_{i}\left(u\left(t_{i}\right)\right)+\sum_{i=1}^{m} G_{3}\left(t, t_{i}\right) I_{i}\left(u\left(t_{i}\right)\right)\right] \mathrm{d} t \\
& \geq \frac{b}{a} \kappa_{1} \int_{0}^{1} f(t, u(t)) M(t) \mathrm{d} t+\frac{b^{2}}{(a-b)^{2}} \kappa_{1} \sum_{i=1}^{m} J_{i}\left(u\left(t_{i}\right)\right)+\frac{b}{a-b} \kappa_{1} \sum_{i=1}^{m} I_{i}\left(u\left(t_{i}\right)\right) .
\end{aligned}
$$

Combining this and (H3), we find

$$
\begin{aligned}
\int_{0}^{1} u(t) M(t) \mathrm{d} t & \geq \frac{b}{a} \kappa_{1} \int_{0}^{1} M(t)\left(a_{1} u(t)-c\right) \mathrm{d} t+\frac{b^{2}}{(a-b)^{2}} \kappa_{1} \sum_{i=1}^{m}\left(a_{3} u\left(t_{i}\right)-c\right)+\frac{b}{a-b} \kappa_{1} \sum_{i=1}^{m}\left(a_{2} u\left(t_{i}\right)-c\right) \\
& =\frac{b}{a} a_{1} \kappa_{1} \int_{0}^{1} u(t) M(t) \mathrm{d} t+\frac{b^{2}}{(a-b)^{2}} a_{3} \kappa_{1} \sum_{i=1}^{m} u\left(t_{i}\right)+\frac{b}{a-b} a_{2} \kappa_{1} \sum_{i=1}^{m} u\left(t_{i}\right)-c_{1},
\end{aligned}
$$

where $c_{1}=\frac{b c}{a} \kappa_{1}^{2}+\frac{b^{2} c m}{(a-b)^{2}} \kappa_{1}+\frac{b c m}{a-b} \kappa_{1}$. Next we consider the following two cases.

Case 1. $\frac{b}{a} a_{1} \kappa_{1} \geq 1$. From 3.5 and $u \in P_{0}$, we obtain

$$
\begin{aligned}
c_{1} & \geq\left(\frac{b}{a} a_{1} \kappa_{1}-1\right) \int_{0}^{1} u(t) M(t) \mathrm{d} t+\frac{b^{2}}{(a-b)^{2}} a_{3} \kappa_{1} \sum_{i=1}^{m} u\left(t_{i}\right)+\frac{b}{a-b} a_{2} \kappa_{1} \sum_{i=1}^{m} u\left(t_{i}\right) \\
& \geq\left(\frac{b}{a} a_{1} \kappa_{1}-1\right) \int_{0}^{1} \frac{b^{2}}{a^{2}}\|u\| M(t) \mathrm{d} t+\frac{b^{2}}{(a-b)^{2}} a_{3} \kappa_{1} \sum_{i=1}^{m} \frac{b^{2}}{a^{2}}\|u\|+\frac{b}{a-b} a_{2} \kappa_{1} \sum_{i=1}^{m} \frac{b^{2}}{a^{2}}\|u\| .
\end{aligned}
$$

This shows that there exists $M_{2}>0$ such that

$$
\|u\| \leq \frac{a^{2}(a-b)^{2}}{b^{2}} \cdot \frac{c_{1}}{a_{3} b^{2} \kappa_{1} m+a_{2} b(a-b) \kappa_{1} m+\kappa_{1}(a-b)^{2}\left(\frac{b}{a} a_{1} \kappa_{1}-1\right)}:=M_{2} \text {, for all } u \in \mathcal{M}_{1} .
$$


Case 2. $\frac{b}{a} a_{1} \kappa_{1}<1$. From 3.5 , we have

$$
c_{1}+\left(1-\frac{b}{a} a_{1} \kappa_{1}\right) \int_{0}^{1} u(t) M(t) \mathrm{d} t \geq \frac{b^{2}}{(a-b)^{2}} a_{3} \kappa_{1} \sum_{i=1}^{m} u\left(t_{i}\right)+\frac{b}{a-b} a_{2} \kappa_{1} \sum_{i=1}^{m} u\left(t_{i}\right) .
$$

Note that $u \in P_{0}$, we have

$$
c_{1}+\left(1-\frac{b}{a} a_{1} \kappa_{1}\right) \kappa_{1}\|u\| \geq \frac{b^{2}}{(a-b)^{2}} a_{3} \kappa_{1} \sum_{i=1}^{m} \frac{b^{2}}{a^{2}}\|u\|+\frac{b}{a-b} a_{2} \kappa_{1} \sum_{i=1}^{m} \frac{b^{2}}{a^{2}}\|u\| .
$$

Therefore,

$$
\|u\| \leq \frac{c_{1} a^{2}(a-b)^{2}}{a_{3} b^{4} \kappa_{1} m+a_{2} b^{3}(a-b) \kappa_{1} m-(a-b)^{2}\left(a^{2}-a b a_{1} \kappa_{1}\right) \kappa_{1}}=: M_{3}, \quad \text { for all } u \in \mathcal{M}_{1} .
$$

To sum up, $\mathcal{M}_{1}$ is a bounded set, as required. Taking $R>\max \left\{M_{2}, M_{3}\right\}$, we obtain

$$
u \neq \mathcal{A} u+\lambda \psi, \text { for all } u \in \partial B_{R} \cap P, \quad \lambda \geq 0 .
$$

Lemma 2.8 yields

$$
i\left(\mathcal{A}, B_{R} \cap P, P\right)=0 .
$$

Let $\mathcal{M}_{2}:=\left\{u \in \bar{B}_{r} \cap P: u=\lambda \mathcal{A} u, \lambda \in[0,1]\right\}$. We shall prove $\mathcal{M}_{2}=\{0\}$. Indeed, if $u \in \mathcal{M}_{2}$, we have $u \in P_{0}$ and

$$
u(t) \leq \int_{0}^{1} G_{1}(t, s) f(s, u(s)) \mathrm{d} s+\sum_{i=1}^{m} G_{2}\left(t, t_{i}\right) J_{i}\left(u\left(t_{i}\right)\right)+\sum_{i=1}^{m} G_{3}\left(t, t_{i}\right) I_{i}\left(u\left(t_{i}\right)\right), \text { for all } u \in \bar{B}_{r} \cap P .
$$

Similar to (3.4), multiplying by $M(t)$ on both sides of the above and integrating over $[0,1]$, we obtain

$$
\begin{aligned}
\int_{0}^{1} u(t) M(t) \mathrm{d} t \leq & \kappa_{1} \int_{0}^{1} M(t) f(t, u(t)) \mathrm{d} t+\frac{a^{2}}{(a-b)^{2}} \kappa_{1} \sum_{i=1}^{m} J_{i}\left(u\left(t_{i}\right)\right) \\
& +\frac{a}{a-b} \kappa_{1} \sum_{i=1}^{m} I_{i}\left(u\left(t_{i}\right)\right), \text { for all } u \in \bar{B}_{r} \cap P .
\end{aligned}
$$

This, together with (H4), implies that

$$
\int_{0}^{1} u(t) M(t) \mathrm{d} t \leq \kappa_{1} b_{1} \int_{0}^{1} u(t) M(t) \mathrm{d} t+\frac{a^{2}}{(a-b)^{2}} b_{3} \kappa_{1} \sum_{i=1}^{m} u\left(t_{i}\right)+\frac{a}{a-b} b_{2} \kappa_{1} \sum_{i=1}^{m} u\left(t_{i}\right) .
$$

From $u \in P_{0}$ we have

$$
\frac{\left(1-\kappa_{1} b_{1}\right) b^{2}}{a^{2}} \kappa_{1}\|u\| \leq\left(1-\kappa_{1} b_{1}\right) \int_{0}^{1} u(t) M(t) \mathrm{d} t \leq \frac{a^{2}}{(a-b)^{2}} b_{3} \kappa_{1} \sum_{i=1}^{m}\|u\|+\frac{a}{a-b} b_{2} \kappa_{1} \sum_{i=1}^{m}\|u\|,
$$

which contradicts the condition $\frac{\left(1-\kappa_{1} b_{1}\right) b^{2}}{a^{2}} \kappa_{1}>m\left[\frac{a^{2}}{(a-b)^{2}} b_{3} \kappa_{1}+\frac{a}{a-b} b_{2} \kappa_{1}\right]$. This implies $\mathcal{M}_{2}=\{0\}$ and thus $u \neq \lambda \mathcal{A} u$ for all $u \in \partial B_{r} \cap P$ and $\lambda \in[0,1]$. Lemma 2.9 yields

$$
i\left(\mathcal{A}, B_{r} \cap P, P\right)=1 .
$$

Equations 3.9 and 3.12 imply that

$$
i\left(\mathcal{A},\left(B_{R} \backslash \bar{B}_{r}\right) \cap P, P\right)=0-1=-1 .
$$

Hence the operator $\mathcal{A}$ has at least one fixed point on $\left(B_{R} \backslash \bar{B}_{r}\right) \cap P$ and therefore (1.1) has at least one positive solution. This completes the proof. 
Theorem 3.3. Suppose that (H1), (H5) and (H6) hold. Then (1.1) has at least one positive solution.

Proof. Let $\mathcal{M}_{3}:=\left\{u \in \bar{B}_{r} \cap P: u=\mathcal{A} u+\lambda \psi, \lambda \geq 0\right\}$, where $\psi \in P_{0}$ is a given element. We claim $\mathcal{M}_{3} \subset\{0\}$. Indeed, if $u \in \mathcal{M}_{3}$, then $u \in P_{0}$ and $u \geq \mathcal{A} u$. By (H5) and (3.5), we have

$$
\int_{0}^{1} u(t) M(t) \mathrm{d} t \geq \frac{b}{a} a_{4} \kappa_{1} \int_{0}^{1} u(t) M(t) \mathrm{d} t+\frac{b^{2}}{(a-b)^{2}} a_{6} \kappa_{1} \sum_{i=1}^{m} u\left(t_{i}\right)+\frac{b}{a-b} a_{5} \kappa_{1} \sum_{i=1}^{m} u\left(t_{i}\right) .
$$

If $\frac{b}{a} a_{4} \kappa_{1} \geq 1$, note that $u \in P_{0}$, then

$$
\begin{aligned}
0 & \geq\left(\frac{b}{a} a_{4} \kappa_{1}-1\right) \int_{0}^{1} u(t) M(t) \mathrm{d} t+\frac{b^{2}}{(a-b)^{2}} a_{6} \kappa_{1} \sum_{i=1}^{m} u\left(t_{i}\right)+\frac{b}{a-b} a_{5} \kappa_{1} \sum_{i=1}^{m} u\left(t_{i}\right) \\
& \geq\left(\frac{b}{a} a_{4} \kappa_{1}-1\right) \int_{0}^{1} \frac{b^{2}}{a^{2}}\|u\| M(t) \mathrm{d} t+\frac{b^{2}}{(a-b)^{2}} a_{6} \kappa_{1} \sum_{i=1}^{m} \frac{b^{2}}{a^{2}}\|u\|+\frac{b}{a-b} a_{5} \kappa_{1} \sum_{i=1}^{m} \frac{b^{2}}{a^{2}}\|u\| .
\end{aligned}
$$

This shows $\|u\| \equiv 0, \forall u \in \mathcal{M}_{3}$.

If $\frac{b}{a} a_{4} \kappa_{1}<1$, then

$$
\begin{aligned}
\left(1-\frac{b}{a} a_{4} \kappa_{1}\right) \kappa_{1}\|u\| & \geq\left(1-\frac{b}{a} a_{4} \kappa_{1}\right) \int_{0}^{1} u(t) M(t) \mathrm{d} t \geq \frac{b^{2}}{(a-b)^{2}} a_{6} \kappa_{1} \sum_{i=1}^{m} u\left(t_{i}\right)+\frac{b}{a-b} a_{5} \kappa_{1} \sum_{i=1}^{m} u\left(t_{i}\right) \\
& \geq \frac{b^{2}}{(a-b)^{2}} a_{6} \kappa_{1} \sum_{i=1}^{m} \frac{b^{2}}{a^{2}}\|u\|+\frac{b}{a-b} a_{5} \kappa_{1} \sum_{i=1}^{m} \frac{b^{2}}{a^{2}}\|u\|,
\end{aligned}
$$

which contradicts the property $\left(1-\frac{b}{a} a_{4} \kappa_{1}\right) \kappa_{1}<\kappa_{1} m\left[\frac{b^{2}}{(a-b)^{2}} a_{6} \frac{b^{2}}{a^{2}}+\frac{b}{a-b} a_{5} \frac{b^{2}}{a^{2}}\right]$. This also verify $\|u\| \equiv 0, \forall u \in$ $\mathcal{M}_{3}$.

Hence $\mathcal{M}_{3} \subset\{0\}$, as claimed. As a result, we have $u-\mathcal{A} u \neq \lambda \psi$ for all $u \in \partial B_{r} \cap P$ and $\lambda \geq 0$. Lemma 2.8 gives

$$
i\left(\mathcal{A}, B_{r} \cap P, P\right)=0 .
$$

Let $\mathcal{M}_{4}:=\{u \in P: u=\lambda \mathcal{A} u, \lambda \in[0,1]\}$. We assert $\mathcal{M}_{4}$ is bounded. Indeed, if $u \in \mathcal{M}_{4}$, then we have $u \in P_{0}$ and $u \leq \mathcal{A} u$, which can be written in the form

$$
u(t) \leq \int_{0}^{1} G_{1}(t, s) f(s, u(s)) \mathrm{d} s+\sum_{i=1}^{m} G_{2}\left(t, t_{i}\right) J_{i}\left(u\left(t_{i}\right)\right)+\sum_{i=1}^{m} G_{3}\left(t, t_{i}\right) I_{i}\left(u\left(t_{i}\right)\right) .
$$

By (H6) and (3.10), we obtain

$$
\int_{0}^{1} u(t) M(t) \mathrm{d} t \leq b_{4} \kappa_{1} \int_{0}^{1} u(t) M(t) \mathrm{d} t+\frac{a^{2}}{(a-b)^{2}} b_{6} \kappa_{1} \sum_{i=1}^{m} u\left(t_{i}\right)+\frac{a}{a-b} b_{5} \kappa_{1} \sum_{i=1}^{m} u\left(t_{i}\right)+c_{2},
$$

where $c_{2}=\kappa_{1}^{2} c+\frac{a^{2} c m}{(a-b)^{2}} \kappa_{1}+\frac{a c m}{a-b} \kappa_{1}$.

From $u \in P_{0}$, we get

$$
\begin{aligned}
\frac{\left(1-b_{4} \kappa_{1}\right) b^{2}}{a^{2}} \kappa_{1}\|u\| & \leq\left(1-b_{4} \kappa_{1}\right) \int_{0}^{1} u(t) M(t) \mathrm{d} t \leq \frac{a^{2}}{(a-b)^{2}} b_{6} \kappa_{1} \sum_{i=1}^{m} u\left(t_{i}\right)+\frac{a}{a-b} b_{5} \kappa_{1} \sum_{i=1}^{m} u\left(t_{i}\right)+c_{2} \\
& \leq \frac{a^{2}}{(a-b)^{2}} b_{6} \kappa_{1} \sum_{i=1}^{m}\|u\|+\frac{a}{a-b} b_{5} \kappa_{1} \sum_{i=1}^{m}\|u\|+c_{2} .
\end{aligned}
$$


Consequently, we see

$$
\|u\| \leq \frac{c_{2} a^{2}(a-b)^{2}}{\left(1-\kappa_{1} b_{4}\right) b^{2}(a-b)^{2} \kappa_{1}-\kappa_{1} m\left[a^{4} b_{6}+a^{3}(a-b) b_{5}\right]}:=M_{4} .
$$

Now $\mathcal{M}_{4}$ is a bounded set, as asserted. Taking $R>M_{4}$, we have $u \neq \lambda \mathcal{A} u$ for all $u \in \partial B_{R} \cap P$ and $\lambda \in[0,1]$. Lemma 2.9 yields

$$
i\left(\mathcal{A}, B_{R} \cap P, P\right)=1 .
$$

Equations 3.14 and 3.15 imply that

$$
i\left(\mathcal{A},\left(B_{R} \backslash \bar{B}_{r}\right) \cap P, P\right)=1-0=1 .
$$

Hence the operator $\mathcal{A}$ has at least one fixed point on $\left(B_{R} \backslash \bar{B}_{r}\right) \cap P$ and therefore, 1.1 has at least one positive solution. This completes the proof.

\section{Acknowledgment}

This paper is supported by Shandong Provincial Natural Science Foundation (ZR2015AM014); China Postdoctoral Science Foundation(2015M582070); Shandong Province Postdoctoral Innovation Project Special Foundation(201502022); Major Project of Qilu Normal University(2015ZDL01).

\section{References}

[1] B. Ahmad, S. Sivasundaram, Existence results for nonlinear impulsive hybrid boundary value problems involving fractional differential equations, Nonlinear Anal. Hybrid Syst., 3 (2009), 251-258. 1

[2] B. Ahmad, S. Sivasundaram, Existence of solutions for impulsive integral boundary value problems of fractional order, Nonlinear Anal. Hybrid Syst., 4 (2010), 134-141.

[3] A. Anguraj, M. Kasthuri, P. Karthikeyan, Integral boundary value problems for fractional impulsive integro differential equations in Banach spaces, Int. J. Anal. Appl., 5 (2014), 56-67.

[4] A. Bouzaroura, S. Mazouzi, Existence results for certain multi-orders impulsive fractional boundary value problem, Results Math., 66 (2014), 1-20.

[5] Y. Chen, Z. Lv, Z. Xu, Solvability for an impulsive fractional multi-point boundary value problem at resonance, Bound. Value Probl., 2014 (2014), 14 pages. 1

[6] D. Guo, V. Lakshmikantham, Nonlinear Problems in Abstract Cones, Academic Press, New York, (1988). 2.8 2.9

[7] L. Hu, L. Liu, Y. Wu, Positive solutions of nonlinear singular two-point boundary value problems for second-order impulsive differential equations, Appl. Math. Comput., 196 (2008), 550-562. 1

[8] M. Krasnoselskii, P. Zabreiko, Geometrical methods of nonlinear analysis, Springer-Verlag, New York, (1984). 2.10

[9] X. Li, F. Chen, X. Li, Generalized anti-periodic boundary value problems of impulsive fractional differential equations, Commun. Nonlinear Sci. Numer. Simul., 18 (2013), 28-41. 1

[10] X. Liu, M. Jia, Existence of solutions for the integral boundary value problems of fractional order impulsive differential equations, Math. Methods Appl. Sci., 39 (2016), 475-487. 1

[11] Z. Liu, X. Li, Existence and uniqueness of solutions for the nonlinear impulsive fractional differential equations, Commun. Nonlinear Sci. Numer. Simul., 18 (2013), 1362-1373. 1

[12] I. Podlubny, Fractional differential equations: an introduction to fractional derivatives, fractional differential equations, to methods of their solution and some of their applications, Academic Press, San Diego - New York London, (1999). 2

[13] H. M. Srivastava, J. J. Trujillo, Theory and applications of fractional differential equations, Elsevier Science, Amsterdam, The Netherlands, (2006). 2

[14] G. Wang, B. Ahmad, L. Zhang, Some existence results for impulsive nonlinear fractional differential equations with mixed boundary conditions, Comput. Math. Appl., 62 (2011), 1389-1397. 1

[15] G. Wang, B. Ahmad, L. Zhang, Impulsive anti-periodic boundary value problem for nonlinear differential equations of fractional order, Nonlinear Anal., 74 (2011), 792-804. 1

[16] W. Wang, X. Fu, X. Yang, Positive solutions of periodic boundary value problems for impulsive differential equations, Comput. Math. Appl., 58 (2009), 1623-1630. 1

[17] X. Zhang, L. Liu, Y. Wu, Existence results for multiple positive solutions of nonlinear higher order perturbed fractional differential equations with derivatives, Appl. Math. Comput., 219 (2012), 1420-1433. 1 
[18] X. Zhang, L. Liu, Y. Wu, B. Wiwatanapataphee, The spectral analysis for a singular fractional differential equation with a signed measure, Appl. Math. Comput., 257 (2015), 252-263.

[19] X. Zhang, Y. Wu, L. Caccetta, Nonlocal fractional order differential equations with changing-sign singular perturbation, Appl. Math. Model., 39 (2015), 6543-6552. 1

[20] K. Zhang, J. Xu, W. Dong, Positive solutions for a fourth-order p-Laplacian boundary value problem with impulsive effects, Bound. Value Probl., 2013 (2013), 12 pages. 1

[21] K. Zhao, Multiple positive solutions of integral BVPs for high-order nonlinear fractional differential equations with impulses and distributed delays, Dyn. Syst., 30 (2015), 208-223. 1

[22] K. Zhao, P. Gong, Positive solutions for impulsive fractional differential equations with generalized periodic boundary value conditions, Adv. Dierence Equ., 2014 (2014), 19 pages. 2.5. 2.6

[23] J. Zhou, M. Feng, Green's function for Sturm-Liouville-type boundary value problems of fractional order impulsive differential equations and its application, Bound. Value Probl., 2014 (2014), 21 pages. 1 